03,09

\title{
Экситон-фононное стимулированное излучение в кристаллической пленке ZnO при комнатной температуре
}

\author{
(ㄱ Н.Н. Васильев, Е.Н. Борисов, Б.В. Новиков \\ Санкт-Петербургский государственный университет, \\ Санкт-Петербург, Россия \\ E-mail: n.vassiliev@mail.ru
}

Поступила в Редакцию 23 апреля 2020г.

В окончательной редакции 23 апреля 2020 г.

Принята к публикации 28 апреля 2020 г.

\begin{abstract}
Исследована близкраевая люминесценция эпитаксиальной пленки оксида цинка, выращенная молекулярнопучковой эпитаксией на сапфировой подложке. С увеличением оптического возбуждения при комнатной температуре спектр люминесценции радикально меняется и возникает новая полоса с максимумом $\sim 3.17 \mathrm{eV}$. Она имеет свойства стимулированного излучения: порога нелинейного роста и сужения полуширины. C помощью модели одномерного усилителя и экспериментальных данных расчитан спектр усиления, который имеет максимальное значение $\sim 170 \mathrm{~cm}^{-1}$. Проведен анализ теоретических подходов для вычисления концентрации Мотта. Впервые показано, что вблизи пороговой интенсивности наблюдаемое стимулированное излучение развивается на основе второго фононного повторения экситона.
\end{abstract}

Ключевые слова: пленка $\mathrm{ZnO}$, усиление, экситонный механизм.

DOI: 10.21883/FTT.2020.10.49904.098

\section{1. Введение}

Интерес к бинарному полупроводнику оксида цинка $(\mathrm{ZnO})$ возродился в связи с новыми возможностями нанотехнологий и его большими потенциальными возможностями для применения в оптоэлектронике. Ширина его запрещенной зоны составляет $\sim 3.4 \mathrm{eV}$, энергия связи экситона - $60 \mathrm{meV}$. Это делает его конкурентом другого широкозонного полупроводника $\mathrm{GaN}$ для применений стимулированного излучения (ASE) в ультрафиолетовых (UV) источниках света в виде свето- или лазерных диодов [1,2]. Большая энергия связи и относительная простота технологий роста выгодно отличает $\mathrm{ZnO}$ от $\mathrm{GaN}$ и создает перспективу для использования первого при повышенных температурах.

Необходимым этапом правильного описания и расчетов ASE является определение его природы. Главные механизмы UV ASE в ZnO основаны на близкраевой люминесценции и связаны с экситонами и вырожденной электронно-дырочной плазмой (ЕНР). Экситонный механизм считается более предпочтительным, поскольку в принципе дает меньший порог ASE для интенсивности оптического возбуждения $I_{\text {exc }}$ (накачки) и больший коэффициент усиления $g$. При низких температурах и небольших $I_{\text {exc }}$ преобладает ASE экситонной природы [3]. При комнатной температуре (RT) экситонный и плазменный механизмы часто трудно отличить, т.к. спектры нелинейных полос различной природы сливаются в одну полосу. Идентификация ЕНР визуально возможна, если исследуемый образец не деградирует при достаточно больших $I_{\text {exc }}$. При этом плазменная полоса проявляется с длинноволновой стороны относи- тельно первичной нелинейной полосы. При дальнейшем увеличении $I_{\text {exc }}$ она продолжает отделяться или смещаться в ту же сторону с меньшей скоростью и становится доминантной (см. например [4]). При меньших накачках могут проявляться экситонный и плазменный механизмы в процессах одной и той же физической природы. Это происходит потому, что ASE может быть связана как с экситонами, так и с коррелированными электронно-дырочными парами $(e-h)$. Один из таких механизмов - рассеяние электрона на $e-h$-паре, который можно идентифицировать по характерной температурной зависимости полосы ASE [5-7]. Рассеяние на паре недавно было обнаружено в микрокристаллах и наночастицах $[8,9]$. Другой механизм - рекомбинация $e-h$-пары при взаимодействии с плазмон-фононными модами [10]. Расчет концентрации $e-h$-пар $n_{e h}$ не может быть единственно надежным основанием для идентификации полос. Во-первых, время жизни нелинейной полосы, входящее в расчет, часто известно не точно. Вовторых, используемое значение концентрации Мотта $n_{M}$ зависит от теоретического подхода.

Переход от изучения ASE в объемных монокристаллах $\mathrm{ZnO}$ к нанокристаллическим пленкам выявил большие различия в рабочих интенсивностях $I_{\text {exc }}$ и коэффициентах $g$. Если в монокристаллах масштаб накачек $I_{\text {exc }} \sim 10^{7}-10^{8} \mathrm{~W} / \mathrm{cm}^{2}[11]$, то в пленках он снизился приблизительно на два порядка [2]. Однако значения $g \sim 10^{3}-10^{4} \mathrm{~cm}^{-1}$, обычно получаемые в монокристаллах при низких температурах, оказались не досягаемы в пленках (см. например [12,13]). В то же время, при RT в пленках убедительно достигнуты значения $g$ порядка $10^{2} \mathrm{~cm}^{-1}$, которых в монокристаллах получить 
не удалось. Анонсированные очень высокие значения $g \sim 10^{3} \mathrm{~cm}^{-1}$ в пленках, выращенных осаждением с помощью импульсного лазера и молекулярно-пучковой эпитаксией (MBE), на наш взгляд, нуждаются в подтверждениями дополнительными данными [14,15]. Так, при больших усилениях начальный участок зависимости интенсивности люминесценции $\ln I_{l u m}$ от длины усиления очень крутой. Поэтому необходимы данные наклона этой зависимости от накачки $I_{\text {exc }}$ и длин волн [11]. С целью получить больше сведений о роли экситонов в механизмах и параметрах ASE предринято настоящее исследование на пленках, выращенных MBE.

\section{2. Экспериментальные условия}

Образец выращивался традиционным методом МВЕ на сапфире без последующего отжига. Спектры люминесценции образца регистрировались двойным монохроматором MDR-6 с апертурой $1: 6$, фотоумножителем Hamamatsu H7844 с предусилителем SR445A и счетом фотонов SR440. Источником слабого возбужения служил фемтосекундный лазер Mira Optima 900-D (Coherent) с удвоением частоты на длине волны $361.1 \mathrm{~nm}$, частотой повторения $1000 \mathrm{~Hz}$ и средней мощностью $0.018 \mathrm{~mW}$. Сильное возбуждение обеспечивалось лазером Matrix 355 с длительностью импульca $T_{\text {exc }}=7 \mathrm{~ns}$, частотой повторения $200 \mathrm{~Hz}$ на длине волны $354.7 \mathrm{~nm}$. Максимальная интенсивность возбуждения достигала $I_{\text {exc }} \sim 10^{3} \mathrm{~kW} / \mathrm{cm}^{2}$ на прямоугольной площадке (полоске) возбуждения $40 \times 500 \mu \mathrm{m}$. Полоска формировалась одной цилиндрической линзой и стоящей перед ней диафрагмой, служащей для получения более однородного возбуждения. Коэффициент оптического усиления определялся изменением длины полоски с помощью перемещения края щели, расположенной на микрометрической подвижке вблизи плоскости пленки. Исследование кристаллической структуры образца и получение спектров рентгеновской дифракции (XRD) проводились с помощью дифрактометра Bruker D8 DISCOVER с фильтрацией $\mathrm{Cu}\left(K_{\alpha}\right)$-линии; диаметр пятна падающего луча $\sim 500 \mu \mathrm{m}$. Толщина и морфология пленки изучались сканирующим электронным микроскопом (SEM) Zeiss Merlin с приставкой электронной оптики GEMINI-II. Исследования при низких температурах проводились на оптическом криостате замкнутого цикла Montana.

\section{3. Полученные результаты и их обсуждение}

Исследования с помощью SEM при различных увеличениях показало, что образец $\mathrm{ZnO}$ является монолитной пленкой. При этом ее толщина $d \sim 500 \mathrm{~nm}$. Спектры XRD (рис. 1) содержат практически единственную линию оксида цинка $(000 \overline{2})$ и линию сапфировой подложки $(000 \overline{6})$. Это говорит о строгой ориен-

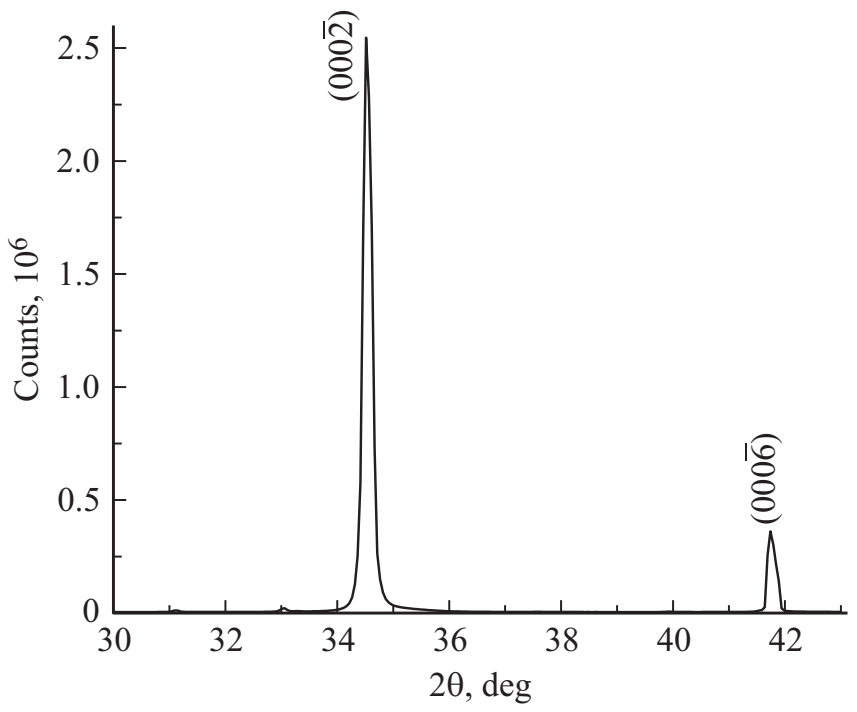

Рис. 1. Фрагмент спектра рентгеновской дифракции образца в области заметного сигнала.

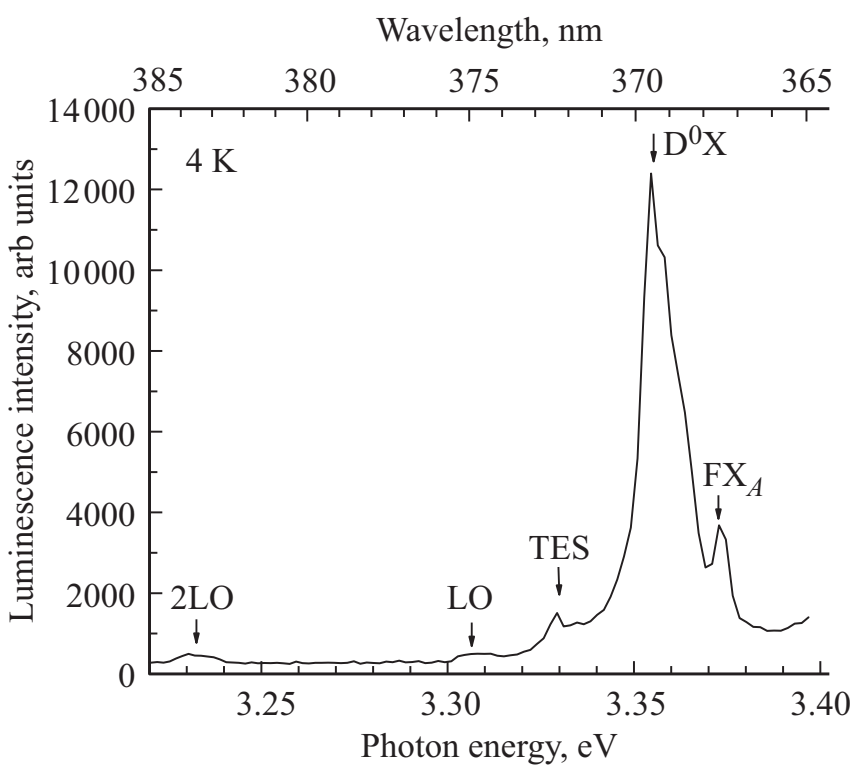

Рис. 2. Спектр близкраевой дюминесценции образца, полученный при $4 \mathrm{~K}$. FX $\mathrm{A}$ - свободный А-экситон. Сильная линия $\mathrm{D}^{0} \mathrm{X}$-полоса, особенность в области $3.32-3.33 \mathrm{eV}$ относится к двухэлектронным саттелитам (TES), LO и 2LO - первое и второе фононные повторения свободного экситона.

тации $c$-оси кристаллической структуры пленки $\mathrm{ZnO}$ нормально ее плоскости. Низкотемператупный спектр, полученный при слабом возбуждении и $4 \mathrm{~K}$, в области экситонной люминесценции содержит хорошо видимый свободный А-экситон $\mathrm{FX}_{\mathrm{A}}(3.373 \mathrm{eV})$ и его фононные повторения LO и $2 \mathrm{LO}$ с энергиями $\sim 3.31$ и $3.23 \mathrm{eV}$ соответственно. Это свидетельствует о высоком качестве образца (рис. 2). В области $\sim 3.35-3.36 \mathrm{eV}$ можно видеть характерную $D^{0} X$-полосу экситонно-примесного комплекса, содержащую несколько слившихся линий 

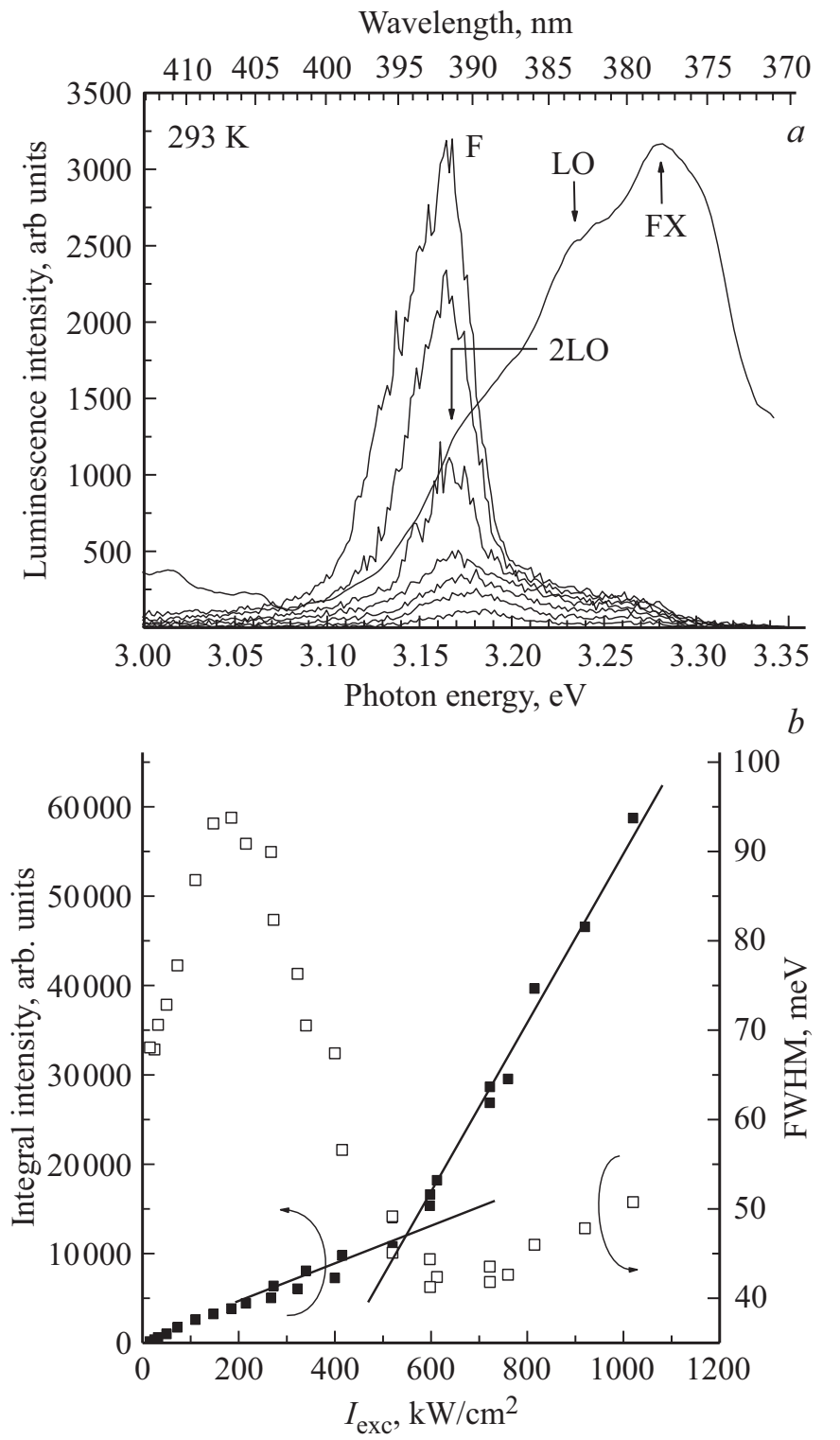

Рис. 3. a) Зависимость спектров люминесценции от интенсивности возбуждения $I_{e x c}$ в единицах пороговой интенсивности $I_{t h}=550 \mathrm{~kW} / \mathrm{cm}^{2}$ (см. ниже) при комнатной температуре: 0.17 , $0.3,0.5,0.7,0.8,1.1,1.5$ считая снизу вверх. Спектр в коротковолновой области получен при слабом возбуждении и нормирован на максимум спектра при интенсивности $I_{e x c}=1.5 I_{t h}$ для удобства сравнения. $\mathrm{F}$ - обозначение появившейся полосы. $b$ ) Зависимость интегральной интенсивности люминесцеции и полуширины усиливаемой полосы от $I_{\text {exc }}$ (темные и светлые квадраты соответственно); излом аппроксимирующих прямых линий показывает порог стимулированного излучения.

связанных на донорах экситонов [16,17]. Относительно слабая полоса в области $\leq 3.33 \mathrm{eV}$ идентифицируется как рекомбинация двухэлектроных сателлитов [18]. На рис. 3, $a$ показаны спектры люминесцеции образца в экситонной области, полученных при RT и различных $I_{\text {exc }}$ лазером Matrix 355. Регистрация сигналов велась с торца образца нормально его боковой грани и длин- ной оси прямоугольника возбужденной полоски. Можно видеть радикальную трансформацию спектров с увеличением $I_{\text {exc }}$ и появление новой полосы. В дальнейшем будем называть ее $F$-полосой. Она имеет тенденцию к смещению максимума к 3.15-3.16 eV. Для сравнения приведен спектр, полученный при слабом возбуждении с помощью фемтосекундного лазера и нормальной к поверхности образца регистрации. Максимум в области $3.28 \mathrm{eV}$ соответствует свободному экситону $\mathrm{FX}$, видны также два его первых фононных повторения с энергиями $\sim 3.23$ и $3.17 \mathrm{eV}$. Рис. $3, b$ показывает рост интегрированной люминесценции и изменение полной полуширины (FWHM) F-полосы с повышением накачки $I_{\text {exc }}$ (черные и пустые квадраты соответственно). Ясно виден нелинейный рост и излом интенсивности. При аппроксимации экспериментальных точек прямыми линиями излом приходится на $I_{\text {exc }} \sim 550 \mathrm{~kW} / \mathrm{cm}^{2}$. FWHM показывает спад, окончание которого приходится на то же значение $I_{\text {exc }}$. Из этих данных можно заключить, что $550 \mathrm{~kW} / \mathrm{cm}^{2}$ это пороговое значение $I_{t h}$ начала ASE. Наши измерения показали, что нелинейный рост интенсивности F-полосы развивается со степенью выше квадратичной.

Спектр усиления расчитывался с помощью приближения одномерного усилителя без учета насыщения в методе возбуждения в форме полоски [11]:

$$
I_{\text {lum }}(h v)=\frac{I_{0}}{g}(\exp (g l)-1),
$$

где $l$ - длина полоски, $I_{0}$ - масштабный множитель.

На рис. 4 показаны спектр усиления для $I_{\text {exc }}=800 \mathrm{~kW} / \mathrm{cm}^{2}$. Максимум значения $g \sim 170 \mathrm{~cm}^{-1}$ виден в области $\sim 3.16 \mathrm{eV}$. Асимметрия спектра указывает на тенденцию к усилению в области энергий, где

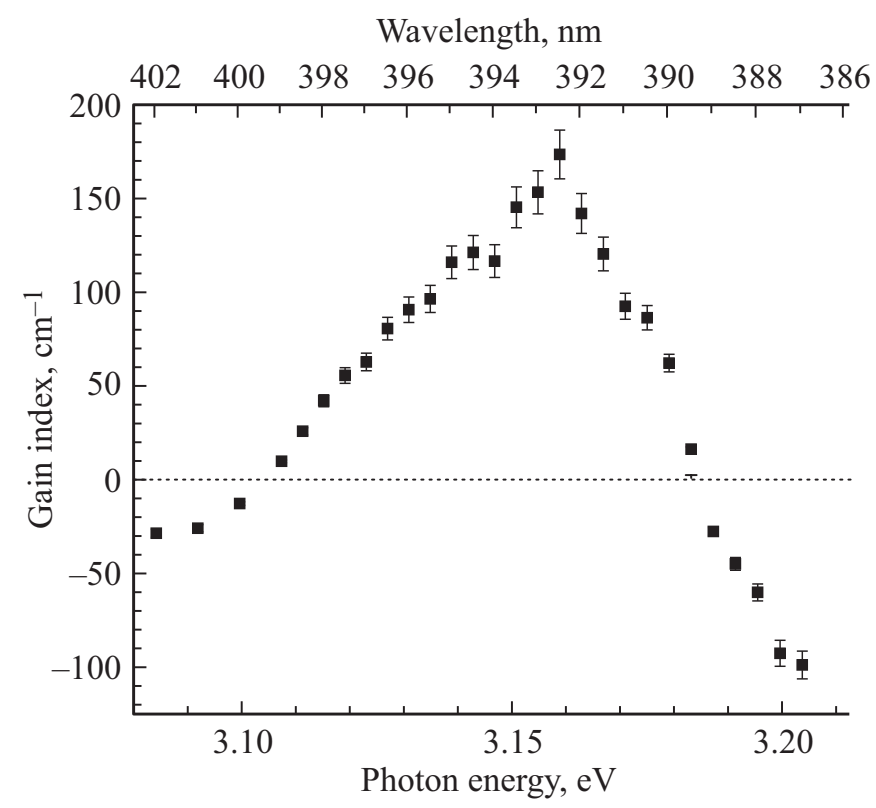

Рис. 4. Спектр усиления для $I_{\text {exc }}=800 \mathrm{~kW} / \mathrm{cm}^{2}$. Пунктирная линия разграничивает области усиления и поглощения. 
поглощение меньше и возможно развитие к усилению с повышением $I_{\text {exc }}$ (см. ниже). Положение максимума характерны для концентраций носителей $e-h$-пар $n_{e h}$, меньшей концентрации Мотта $n_{M}$. Коэффициенты усиления, полученные на пленках MBE при гелиевых температурах, имеют значения $\sim 300 \mathrm{~cm}^{-1}$ (см. например, [12,13]). Полученное в нашем эксперименте значение $g$ свидетельствует об относительно небольшом его уменьшении при RT. Анализ работ показал, что это свойство, вероятно, является общим для наноструктур и выгодно отличает их от объемных монокристаллов.

Чтобы понять природу новой полосы, оценим концентрацию электронно-дырочных пар $n_{e h}$ с помощью формулы [10]:

$$
n_{e h}=I_{e x c} \tau / h \nu l,
$$

где $\tau$ - время жизни люминесценции для спонтанного излучения, если $\tau<T_{\text {exc }}$ (квазинепрерывный режим); $h v=3.49 \mathrm{eV}$ - энергия возбуждающего кванта; $l$ эффективная обратная длина поглощения. Формула (2) является обобщением обычной формулы и справедлива для наноструктур. В (2) коэффициент поглощения заменен на множиттель $1 / l$, учитывающий диффузию носителей. В случае $\mathrm{ZnO}$ длина диффузии $(1-3 \mu \mathrm{m}) \gg d[10]$. Тогда возбуждение проникает на всю толщину пленки, поэтому $l=d$. При RT, нижний предел времени жизни люминесценции $\mathrm{ZnO}$ для не отожженных при температурах $\geq 950^{\circ} \mathrm{C}$ эпитаксиальных пленок $\sim 30$ ps [19]. Тогда для порогового возбуждения $I_{\text {exc }}=550 \mathrm{~kW} / \mathrm{cm}^{2}$, $d \sim 0.05 \mathrm{~cm}$ имеем $n_{e h}^{t h}=0.75 \cdot 10^{18} \mathrm{~cm}^{-3}$. При дальнейшем увеличении $I_{\text {exc }}$ соотношение (2) уже применить не просто, т.к. время жизни начнет уменьшаться из-за появления усиления. Численные расчеы показали, что концентрация будет расти относительно слабо [20]. Полученное значение $h_{e h}^{\text {th }}$ в дальнейшем будет сравниваться с концентрацией Мотта $n_{M}$, имея в виду слабый рост $n_{e h}$ после достижения порога усиления. В литературе имеется разброс значений $n_{M}$ в зависимости от подхода. Применяемая теория многих тел учитывает то, что с ростом концентрации носителей энергия экситона не меняется. Таким образом, моттовская концентрация достигается, когда сдвиг зоны из-за ренормализации сравнивается с энергией связи экситона. Решение этой задачи с учетом зависимости от температуры дает универсальное для полупроводников значение $n_{M} \sim 1.0 \cdot 10^{18} \mathrm{~cm}^{-3}[21]$. Теоретический анализ суммы обменной и корреляционной энергий также показал, что ренормализация зоны зависит только только от концентрации носителей и почти не зависит от зонных характеристик конкретного полупроводника [22]. Этот подход дает $n_{M} \sim 4.5 \cdot 10^{17} \mathrm{~cm}^{-3}$ и не зависит от температуры. Основываясь на этих теоретических расчетах, было предложено не зависящее от температуры значение $n_{M} \sim 3.0 \cdot 10^{17} \mathrm{~cm}^{-3}[10]$. Однако расчетные данные расходятся с экспериментальными для некоторых полупроводников II-VI, a для $\mathrm{ZnO}$ экспериментальных данных в указанной области концентраций, вероятно, нет [23]. При RT часто применяемое приближение Дебая-Хюккеля (DH) для длины экранирования невырожденных экситонов дает оценку для $\mathrm{ZnO} n_{M} \sim 2.6 \cdot 10^{19} \mathrm{~cm}^{-31}$ Это приближение справедливо при условии, когда средняя потенциальная энергия кулоновского взаимодействия зарядов $\langle V\rangle \ll k_{B} T$ [24]. Наша оценка показывает, что при такой концентрации приближение DH уже не применимо. Действительно, $\langle V\rangle=4.64 \cdot 10^{-29} n_{e h}^{1 / 3} \mathrm{~J} \sim 90 \mathrm{meV}>k_{B} T$ при RT. $\mathrm{C}$ другой стороны, численный квантово-механический расчет задачи экранировки, выполненный в приближении $\mathrm{DH}$, дает $n_{M} \sim 1.2 \cdot 10^{18} \mathrm{~cm}^{-3}$ [25]. Это значение почти совпадает с первым из выше приведенных, при этом $\langle V\rangle \approx K_{B} T$. Поэтому далее в этой работе будет использоваться значение $n_{M}=1.0 \cdot 10^{18} \mathrm{~cm}^{-3}$. Тогда имеем $n_{e h}^{t h}<n_{M}$, и наблюдаемая нелинейная полоса вблизи порога относится к экситонной природе. При $I_{\text {exc }}=800 \mathrm{~kW} / \mathrm{cm}^{2}=1.45 I_{t h}$ возможно сосуществование экситонного механизма и плазменного.

Иногда при оценке концентрации берется значение не спонтанного времени жизни, а уменьшенное из-за усиления более, чем на порядок. В результате, как и в случае некорректного применения приближения DH, получается $n_{e h}<n_{M}$. Природа ASE интерпретируется как экситонная (экситон-экситонное неупругое рассеяние Р-полоса). Энергетическое положение Р-полосы определяется соотношением $h v_{\max } \approx E_{F X}(T)-E_{b}\left(1-1 / n^{2}\right)$ - $(3 / 2) k_{B} T$, где $E_{F X}(T)$ - энергия свободного экситона при температуре $T, E_{b}-$ энергия связи экситона, $k_{B}-$ постоянная Больцмана, $n=2,3, \ldots \infty[26,27]$. В нашем случае максимум полосы люминесценции $E_{F X}(293)=3.284 \mathrm{eV}$ (рис. 3,a), $E_{b}=0.060 \mathrm{eV}$. Тогда для $n=2$ и $n \rightarrow \infty$ имеем $h v_{\max }=3.200$ и $3.186 \mathrm{eV}$ соответственно. Эти значения больше энергии максимума F-полосы $3.17 \mathrm{eV}$ при пороговом возбуждении $I_{\text {exc }}=550 \mathrm{~kW} / \mathrm{cm}^{2}$. При дальнейшем увеличении $I_{\text {exc }}$ максимум смещается далее в длиноволновую сторону. Такой часто используемый расчет использует положение максимума полосы люминесценции, испытавшей реабсорбцию; поэтому максимум сдвинут в сторону низких энергий. В результате расчитанные значения для $n=2$ и $n \rightarrow \infty$ также меньше истинных. Но даже этот использованный нами не вполне корректный подход устраняет экситон-экситонное взаимодействие из дальнейшего рассмотрения. Чтобы получить дополнительную информацию о механизме ASE, нами была измерена температурная зависимость максимума F-полосы и 2LO-реплики свободного экситона при $I_{\text {exc }}=800 \mathrm{~kW} / \mathrm{cm}^{2}$ (рис. 5). Для 2LO-реплики точки, начиная со $170 \mathrm{~K}$ и выше, представляют зависимость для слабого возбуждения. Это сделано для того, чтобы надежно выделить 2LO-реплику. Видно, что кривая F-полосы в интервале 200-293 К мало отличается и немного ниже по энергии относительно

\footnotetext{
${ }^{1}$ При нашем расчете учтено экранирование двумя заряженными подсистемами электронов и дырок с одинаковыми концентрациями.
} 


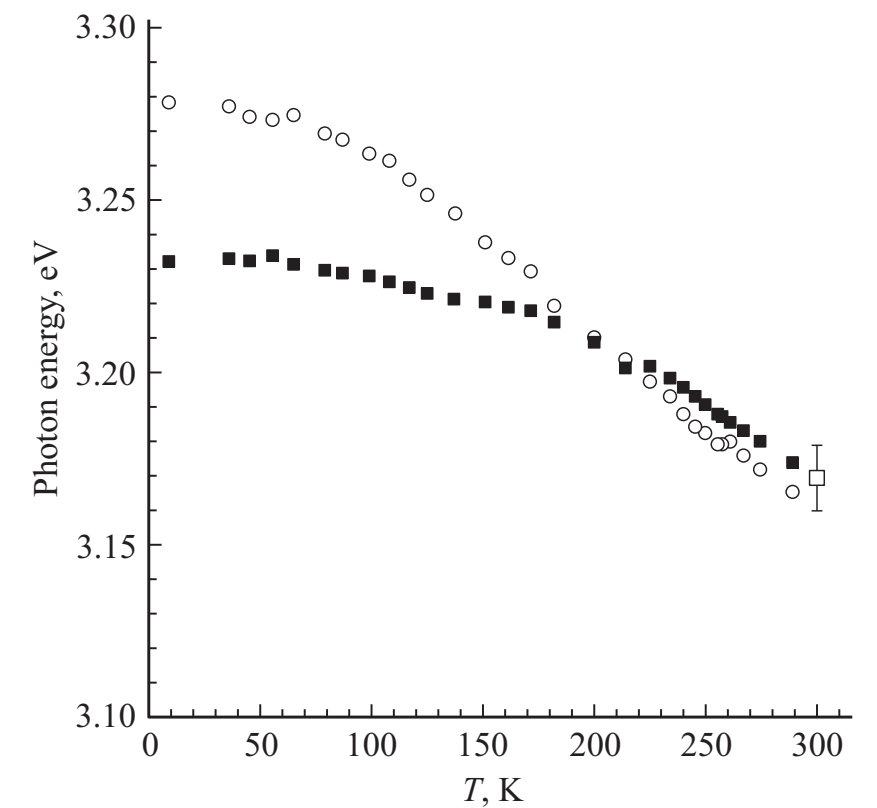

Рис. 5. Температурная зависимость максимумов F-полосы (светлые кружки) и двойного фононного повторения экситона (темные квадраты); интенсивность возбуждения $800 \mathrm{~kW} / \mathrm{cm}^{2}$. Светлый квадрат показывает ошибку второй зависимости.

реплики (см. также рис. $3, a)$. Наши измерения показали, что при накачке вблизи пороговой обе температурные кривые полностью совпадают в интервале $240-300 \mathrm{~K}$. Такое поведение говорит, во-первых, о доминирующем влиянии 2LO-реплики в конкуренци с другими возможными механизмами при RT. Во-вторых, отмеченный небольшой сдвиг является проявлением возникновением нового механизма - плазменного. Это подтверждается длиноволновым крылом на спектре ASE при повышении накачки (рис. $3, a$ ) и заметной деформацией спектра усиления при энергиях, ниже максимума (рис. 4). Таким образом, из наших экспериментов можно заключить, что в нашем образце ASE является результатом рекомбинации экситонов с испусканием двух LO-фононов. $\mathrm{B}$ этой связи F-полосу можно переобозначить на $\mathrm{F}_{2 \mathrm{LO}}$. При больших интенсивностях возбуждения начинает проявляться ЕНР.

\section{4. Заключение}

Исследована близкраевая люминесценция при сильном оптическом возбуждении эпитаксиальной пленки, выращенной молекулярно-пучковой эпитаксией. При увеличении концентрации носителей наблюдалось стимулированное излучение. Оценка концентрации носителей при пороговой интенсивности возбуждения $550 \mathrm{~kW} / \mathrm{cm}^{2}$ дала $0.75 \cdot 10^{18} \mathrm{~cm}^{-3}$. Рассчитан спектр коэффициента усиления при интенсивности возбуждения в 1.5 выше пороговой. Максимальное значение $170 \mathrm{~cm}^{-1}$ получено в области $\sim 3.16 \mathrm{eV}$. При этом концентрация приближается к концентрации Мотта $1.0 \cdot 10^{18} \mathrm{~cm}^{-3}$, и экситонная природа стимулированного излучения переходит в плазменную. Таким образом, измеренный спектр усиления носит двойственный характер сосуществования обоих механизмов. На основе анализа температурных данных впервые показано, что главным механизмом стимулированного излучения в области максимального усиления является рекомбинация экситона с испусканием двух LO-фононов.

\section{Финансирование работы}

Исследования проведены с использованием оборудования ресурсных центров Научного парка СПбГУ „Оптические и лазерные методы исследования вещества“, „Нанотехнологии“ и „Рентгенодифракционные методы исследования“. Образец любезно предоставлен сотрудниками лаборатории С.В. Иванова ФТИ им. Иоффе РАН.

\section{Конфликт интересов}

Авторы заявляют, что у них нет конфликта интересов.

\section{Список литературы}

[1] C. Klingshirn. Phys. Status Solidi B 244, 3027 (2007).

[2] Ü. Özgür, Ya.I. Alivov, C. Liu, A. Teke, M.A. Reshchikov, S. Doan, V. Avrutin, S.-J. Cho, H. Morkoç. J. Appl. Phys. 98, 041301 (2005).

[3] C. Klingshirn. Phys. Status Solidi B 71, 547 (1975).

[4] D.M. Bagnall, Y.F. Chen, Z. Zhu, T. Yao, M.Y. Shen, T. Goto. Appl. Phys. Lett. 73, 1038 (1998).

[5] S. Iwai, S. Namba. Appl. Phys. Lett. 19, 41 (1971).

[6] J.M. Hvam. Phys. Rev. B 4, 4459 (1971).

[7] T. Fischer, J. Bille. J. Appl. Phys. 45, 3937 (1974).

[8] R. Matsuzaki, H. Soma, K. Fukuoka, K. Kodama, A. Asahara, T. Suemoto, Y. Adachi, T. Uchino. Phys. Rev. B 96, 125306 (2017).

[9] N. Vasilyev, E.N. Borisov, B.V. Novikov, I.Kh. Akopyan, M.E. Labzovskaya. J. Lumin. 215, 116668 (2019).

[10] C. Klingshirn, R. Hauschild, J. Fallert, H. Kalt. Phys. Rev. B 75, 115203 (2007).

[11] K.L. Shaklee, R.E. Nahory, R.F. Leheny. J. Lumin. 7, 284 (1973).

[12] H. Priller, J. Brückner, Th. Gruber, C. Klingshirn, H. Kalt, A. Waag, H.J. Ko, T. Yao. Phys. Status Solidi B 241, 587 (2004).

[13] B. Kim, D. Kim, C. Cho, N. Tamura, T. Yamazaki, A. Murayama, K. Kyhm. AIP Conf. Proc. 1399, 25 (2011).

[14] A. Gadallah, K. Nomenyo, C. Couteau, D.J. Rogers, G. Lérondel. Appl. Phys. Lett. 102, 171105 (2013).

[15] Y. Chen, N.T. Tuan, Y. Segawa, Hang-ju Ko, Soon-ku Hong, T. Yao. Appl. Phys. Lett. 78, 1469 (2001).

[16] D.C. Reynolds, D.C. Look, B. Jogai, C.W. Litton, T.C. Collins, W. Harsch, G. Cantwell. Phys. Rev. B 57, 12151 (1998). 
[17] B.K. Meyer, H. Alves, D.M. Hofmann, W. Kriegseis, D. Forster, F. Bertram, J. Christen, A. Hoffmann, M. Straßburg, M. Dworzak, U. Haboeck, A.V. Rodina. Phys. Status Solidi B 241, 231 (2004).

[18] K. Thonke, Th. Gruber, N. Teofilov, R. Schönfelder, A. Waag, R. Sauer. Physica B 308-310, 945 (2001).

[19] Ü.Özgür, A. Teke, C. Liu, S.J. Cho, H. Morkoç, H.O. Everitt. Appl. Phys. Lett. 84, 3223 (2005).

[20] D.S. Wiersma, A. Lagendijk. Phys. Rev. E 544256 (1996).

[21] R. Zimmermann. Phys. Status Solidi B 146, 371 (1988).

[22] P. Vashishta, R.K. Kalia. Phys. Rev. B 25, 6492 (1982).

[23] C. Klingshirn. Semicond. Opt. 3rd ed. Springer, Berlin (2007). P. 534.

[24] P. Debye, E. Hückel. Phys. Z. 24, 185, 305 (1923).

[25] W.A. Albers. Phys. Rev. Lett. 23, 410 (1969).

[26] D. Magde, H. Mahr. Phys. Rev. Lett. 24, 890 (1970).

[27] J.M. Hvam. Solid State Commun. 12, 95 (1973).

Редактор Ю.Э. Китаев 